\title{
Research on the grounding plate effect on the Measurement Precision of the field mill and calibration method
}

\author{
Yan ZhaoWANG ${ }^{1 a}$, Ye MaoZHANG $^{1 \mathrm{~b}}$,Jian GongZHANG ${ }^{1 \mathrm{c}}$, BinZHOU ${ }^{1 \mathrm{~d}}$ \\ ${ }^{1}$ China Electric Power Research Institute Wuhan Branch,State Key Laboratory of Power Grid \\ Environmental Protection \\ ${ }^{\mathrm{a}}$ wangyanzhao@epri.sgcc.com.cn, ${ }^{\mathrm{b}}$ zhangyemao@epri.sgcc.cm.cn, \\ czhangjiangong@epri.sgcc.com.cn, dzhoubing2@epri.sgcc.com.cn.
}

\begin{abstract}
Key words: field mill; grounding plate; electric field; distortion coefficient, calibration Abstract:The field mill is always used to measure the electric field with charge at present. In order to get accurate measurements results, the field mill must be used belong with the ground plate system. The influence of the ground plate area on the measurement result has been researched by the simulation and experiments. The simulation and experiments results showed that the distortion coefficient of the electric field decreases with the increasing of the area of the ground plate. Since when the area of the ground plate has been decreased or canceled, the measurement results must be calibrated. And the calibration method is been proposed.
\end{abstract}

\section{Introduction}

With the increasing of the power transmission by use the FHVDC transmissionlines which possess many advantages over HVAC transmissions lines in economy, system stability and so on. However, the electromagnetic environment of HVDC transmission lines has become a new problem and aroused widespread interest. Because of the corona effect of the wires, the charged particles near the transmissions lines make the electromagnetic environment very complex. Many researchers come from many counties have already carried out a lot of related researches [1 4]. DC electric field strength, as one of the basic electromagnetic parameters of the electromagnetic environment of transmission lines, the measurement accuracy is of great significant.

The Chinese industrial standard points out that the field mill should be used with $1 \mathrm{~m} \times 1 \mathrm{~m}$ metal plate as the ground reference plane, and its reliable grounding. In the actual work, we found that $1 \mathrm{~m}$ $\times 1 \mathrm{~m}$ metal plate volume and weight are very impressive. It is very inconvenient to mobile and transport in the environmental evaluation. While the field mill is used to measure the synthesis of field strength without ground plate, which is researched by some foreign scientific research institutions [5].

The influence of the area of the grounding metal on the measuring results has been quantitatively analyzed. The calibration method without ground plate has been proposed. Some experiments also have been carried out to varied out the calculation results.

\section{Calculation of the effect of the area of the grounding metal plate on the measurement results}

(1)The structure of the field mill and the used parallel plate for the uniform field

The field-mill instrument consists of two parts: a monitor and a probe. Fig 1 shows the schematic diagram and photo of the probe that comprises of two coaxial circular electrodes with several 
identical lobes shaped on them. The size of the probe is: the side length of the cross section $8.8 \mathrm{~cm}$, height $10 \mathrm{~cm}$. The side length of the square metal plate changes in the range of $0.3 \mathrm{~m} \sim 1.5 \mathrm{~m}$, and the thickness is $1 \mathrm{~mm}$. A uniform electric field is generated by a parallel plate which shown in Fig 2 . The diameter of the circular plate is $3 \mathrm{~m}$ and the distance between the plate and the ground is $1 \mathrm{~m}$. The size of the plate and the distance of the two electrodes are big enough to create a uniform electric field the bigger the better. The distortion coefficient $K$ is been used to indicate the electric field distortion. And the distortion factor is equal to the ratio between the maximum value and the average vale of electric field.
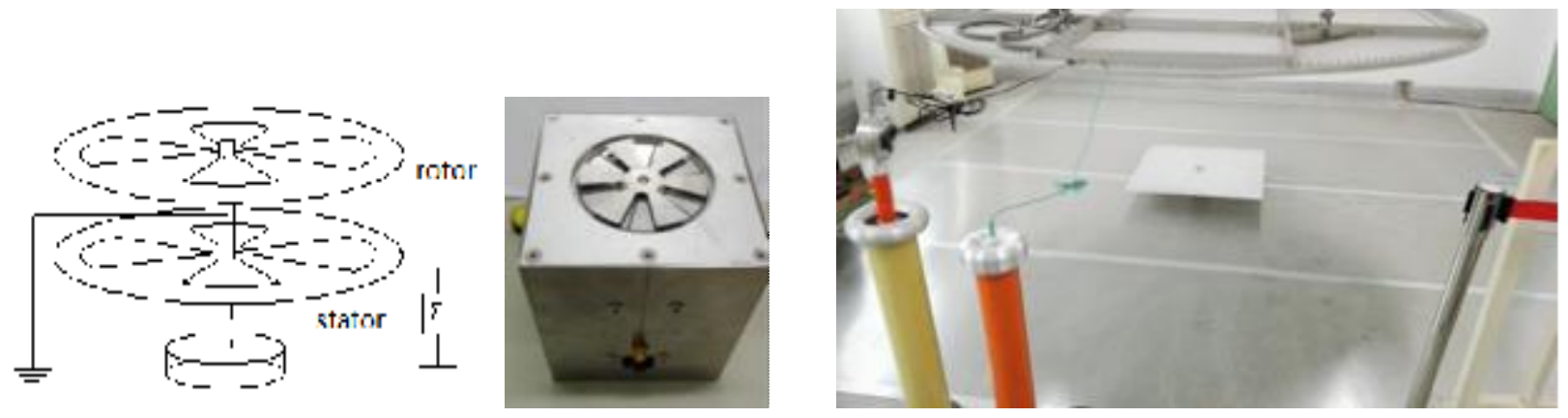

Fig1. Schematic diagram and photo of the field-mill.

(1) With the different area of the ground plate

Fig 2 Photo of the parallel plate

In order to convenience for the engineer applying, one of the methods is to decrease the area of the grounding metal plate, even get rid of it. The influence of the area of the ground plate on the measurement results has been calculated. The electric field distribution is shown in Fig 3. And Fig $3(\mathrm{a})$ is the case of the area of the grounding metal plate is $1 \mathrm{~m}^{2}$, Fig $3(\mathrm{~b})$ is the case of without the grounding metal plate. It seems that the grounding plate increase the electric field because of the decrease of the distance. But the electric field becomes more uniform than that without the grounding metal plate.

The calculation results with different area are shown in Fig 4(a). It seem that the distortion coefficient decreases with the increase of the ground plate area range of $0.3 \mathrm{~m} \sim 1 \mathrm{~m}$. Since then, when the smaller ground plate is used, the field mill should be calibrated with the grounding metal plate for the accuracy measurements. The calibration method will be described in the nest section.

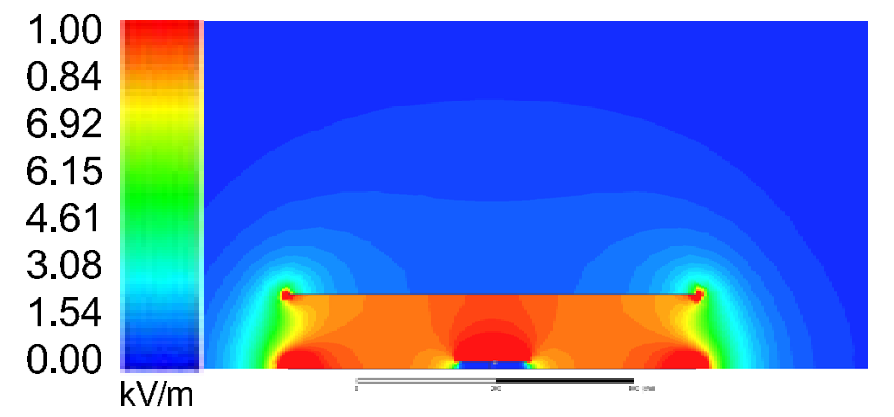

(a) With ground plate;

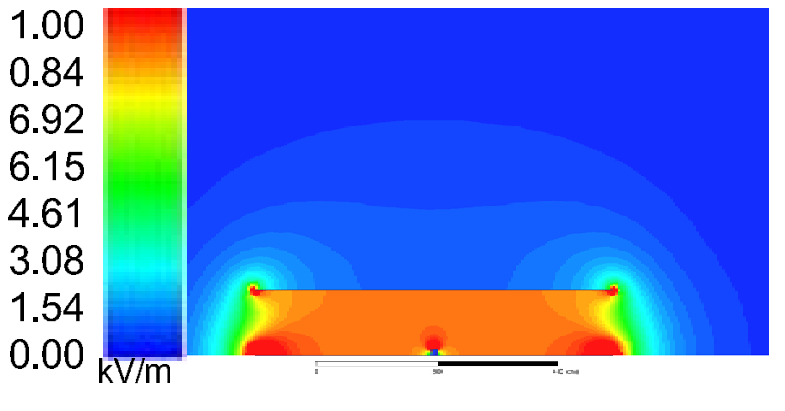

(b) Without ground plate

Fig 3. The electric field distribution at different conditions.

(2) Without the ground plate

The influence of the inter-electrode distance on the distortion factor is shown as Fig 4 (b). It can be seen that the inter-electrode distance has a little influence on the electric field on the top of the field mill. When the probe without metal plate and with metal plate, the distortion factor are 2.36 and1.19 respectively. For the electric field mill without ground plate, the distortion coefficient changed from 2.31 up to 2.45 , and the increase is $6 \%$. In contrast, if the field mill is used with the grounding metal plate, the distance has a little effect on the electric field. Therefore, if the probe 
without the ground plate is used at the outdoor, the measurement result should be calibrated by a constant because of the probe being far away the transmission lines.

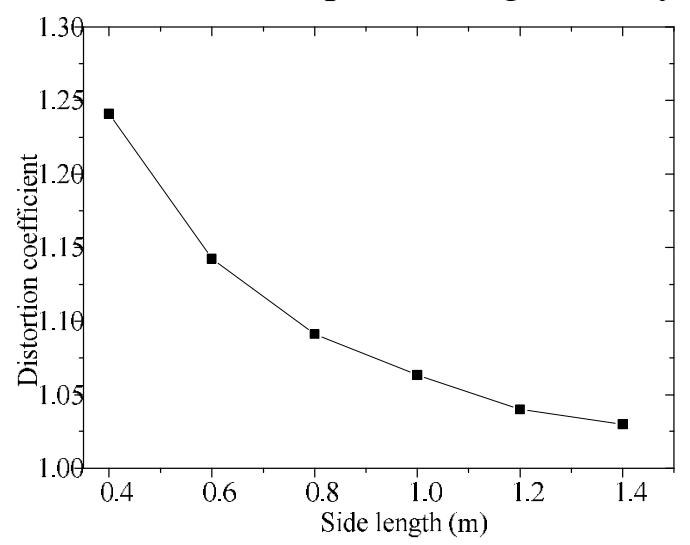

(a) Area of the ground plat;

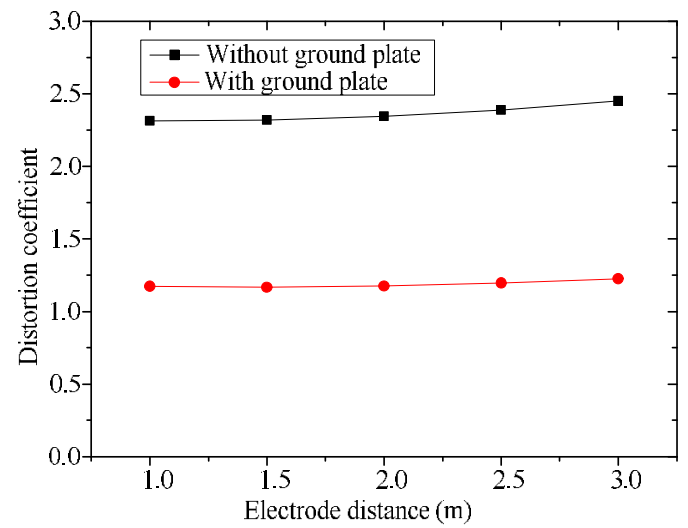

(b) Distance between the electrode and the ground.

Fig 4. The effect factors on the distortion coefficient

\section{Calibration experiments for the field mill with and without the ground plate.}

(1)The field mill is used with the different area of the ground plate.

The influence of the ground plates with different area on the measurements results had been tested on the platform shown in Fig 2. The experiment results are shown in Table 1. In the Table 1, $E_{\text {exp }}$ is the test vale, $E_{\text {tho }}$ is the theory vale calculated though $U / d$. The distortion coefficient $K=E_{\text {exp }} / E_{\text {tho }}$. And the measurement distortion coefficient $K_{l}$ and the simulated distortion coefficient $K_{2}$ are both shown in Table 2. $K_{1}$ is the average vale under different voltage. It can be seen that the changing trend is similar, but the simulated value is smaller than the test value. The reason is that the simulation just considered the influence of structure, but the error of the test value also includes the error component caused by the electronic component in the field mill. However, if the area of the ground plate and the field mill is confirmed, the value of $K$ will be a constant. In this paper, the corrected method can be carried out by the following formula: $E_{\text {cali }}=E_{\text {exp }} / K$ $K$ can use the simulation value. The calibration results are shown in Table 2 by the above method. It can be seen that the calibration results is more close to the theory value.

Table 1 . Test results.

\begin{tabular}{|c|c|c|c|c|c|c|}
\hline \multicolumn{2}{|c|}{$U(\mathrm{kV})$} & 1 & 2 & 3 & $K_{I}$ & $K_{2}$ \\
\hline \multirow{4}{*}{$E_{\text {exp }}(\mathrm{kV} / \mathrm{m})$} & $1 \times 1 \mathrm{~m}$ & 1.21 & 2.38 & 1.20 & 1.08 & 1.06 \\
\cline { 2 - 7 } & $0.8 \times 0.8 \mathrm{~m}$ & 1.24 & 2.49 & 1.24 & 1.12 & 1.09 \\
\cline { 2 - 7 } & $0.65 \times 0.65 \mathrm{~m}$ & 1.32 & 2.61 & 1.31 & 1.18 & 1.12 \\
\cline { 2 - 7 } & $0.34 \times 0.34 \mathrm{~m}$ & 1.53 & 3.02 & 1.52 & 1.37 & 1.28 \\
\hline
\end{tabular}

Table 2 . The calibragted results

\begin{tabular}{|c|c|c|c|c|}
\hline$S$ & $1 \mathrm{~m} \times 1 \mathrm{~m}$ & $0.8 \times 0.8 \mathrm{~m}$ & $0.65 \times 0.65 \mathrm{~m}$ & $0.34 \times 0.34 \mathrm{~m}$ \\
\hline$E_{\text {exp }}(\mathrm{kV} / \mathrm{m})$ & 1.21 & 1.24 & 1.32 & 1.53 \\
\hline$E_{\text {cali }}(\mathrm{kV} / \mathrm{m})$ & 1.14 & 1.14 & 1.18 & 1.19 \\
\hline
\end{tabular}

(2) The field mill is used without the ground plate.

When the ground plate is got rid of, the experiment results are shown in Table 3. $E_{\text {sim }}$ is the simulation value. The distortion coefficient is larger than that without the ground plate. $K_{l}$ decrease with the increase of the voltage. The reason is the test value influenced by other factors such as the instruments error. $K_{2}$ is a constant, which is not change with the voltage. That is why $K_{2}$ is used as 
the calibration coefficient, and the calibration results are shown in Table 4. It seem that the calibration value is close to the theory value, which show that the calibration method is useful.

Table 3. The experiments results without the ground plate.

\begin{tabular}{|c|c|c|c|c|c|}
\hline$U(\mathrm{kV})$ & 1 & 2 & 3 & 4 & 5 \\
\hline$E_{\text {exp }}(\mathrm{kV} / \mathrm{m})$ & 2.53 & 5.12 & 7.65 & 10.55 & 13.24 \\
\hline$K_{1}$ & 2.28 & 2.30 & 2.30 & 2.37 & 2.38 \\
\hline$E_{\text {sim }}(\mathrm{kV} / \mathrm{m})$ & 2.32 & 4.63 & 6.94 & 9.25 & 11.57 \\
\hline$K_{2}$ & 2.32 & 2.31 & 2.31 & 2.31 & 2.31 \\
\hline
\end{tabular}

Table 4 . The calibration results without the ground plate.

\begin{tabular}{|c|c|c|c|c|c|}
\hline$U(\mathrm{kV})$ & 1 & 2 & 3 & 4 & 5 \\
\hline$E_{\text {tho }}(\mathrm{kV} / \mathrm{m})$ & 1.11 & 2.22 & 3.33 & 4.44 & 5.56 \\
\hline$E_{\text {corr }}(\mathrm{kV} / \mathrm{m})$ & 1.10 & 2.22 & 3.31 & 4.57 & 5.73 \\
\hline
\end{tabular}

\section{Conclusions}

Through the above analysis and numerical calculation, the conclusions can be found as following:

1. The ground plate has an impact on the electric field. When the field mill with the different area ground plateis used to measurement the electric field, the field mill must be calibrated with the grounding metal plate. The calibration coefficient can use the distortion coefficient.

2. When the field-mill is used without the ground plate, the measurement results have a large error. Since then the field mill must be calibrated. However, we suggest that the field mill should be used with the grounding metal plate.

\section{Acknowledgements}

This work was financially supported by the Hubei Natural Science Foundation (2015CFA155).

\section{References}

[1] P. SarmaMaruvada, R. D. Dallaire, R. Pedneault, "Development of Field-Mill Instruments for Ground-Level and Above-Ground Electric Field Measurement under HVDC Transmission Lines," IEEETransactions on Power Apparatus and Systems, Vol. PAS-102, No. 3,pp. 738-744, 1983.

[2] Tant, P. , Bolsens, B. , Sels, T. , Van Dommelen, D. , Driesen, J. ,Belmans, R. “ Design and Application of a Field Mill as a High-VoltageDC Meter". Instrumentation and Measurement Technology Conference, 2006. IMTC 2006. Proceedings of the IEEE, Page(s): 1861-1866, 2006.

[3] Abdel-Salam, M. , Al-Hamouz, Z. “ A finite-element analysis of bipolar ionized field ". IEEE Transactions on Vol. 31, Issue: 3, Page(s): 477-483,1995.

[4]Selim, Elsayed O., Waters, Ronald T. " Static Probe for ElectrostaticField Measurement in the Presence of Space Charge “. Industry Applications, IEEE Transactions on Vol. IA-16 , Issue: 3, Page(s): 458-461.

[5]IEEE Guide for the Measurement of DC Electric-Field Strength and IonRelated Quantities, IEEE Standard1227-1990, R2010. 